\title{
Non-Fatal Thrombin Based Hemostatic Matrix Pulmonary Embolism in Laparoscopic Surgery. A Case Report
}

\author{
Mura $\mathrm{P}^{*}$, Fakir S, Mura S, Onida P and Finco G
}

Department of Medical Sciences and Public Health, University of Cagliari, Cagliari, Italy

\begin{abstract}
Locally applied hemostatic agents, are used in various surgical procedures. In our case a thrombin based hemostatic matrix (FloSealTM) was used in the hepatic bed to facilitate haemostasis in a 63 year old-male undergoing video-laparoscopic cholecystectomy. Immediately after the surgery the patient developed severe anxiety, acute shortness of breath with associated hypoxia. An angiographic computerized tomography chest scan demonstrated multiple bilateral sub segmental pulmonary emboli that resolved in the following 12 hours. A bilateral lower extremity duplex ultrasound showed no evidence of deep venous thrombosis.
\end{abstract}

Keywords: Cholecystectomy; Anxiety; Hypoxia; Venous thrombosis; Pulmonary circulation; Hypertension

\section{Introduction}

Thrombin-based hemostatic matrices (TBHM) such as FloSealTM (Baxter, Deerfield, IL, USA) and SurgifoamTM (Ethicon/Johnson \& Johnson Medical, Piscataway, NJ, USA) are gelatin granules mixed with human or bovine thrombin. They are used in a wide variety of surgical procedures to facilitate local hemostasis in addiction to proper surgical technique [1]. Contraindication to intravascular injection of TBHM products is specified on the product labeling information, as thromboembolic events are deemed possible. FloSeal consists of a bovine-derived gelatin matrix with thrombin component made from human plasma. It is aseptic, biocompatible and undergoes readsoption in six to eight weeks. Its widespread clinical diffusion followed immediately its original approvation by the USA Food and Drug Administration in 1999 [1]. The mechanism of FloSeal-induced hemostasis is not restricted to the pathways of coagulation activation, although the contained human thrombin activates the factors V, VIII, and XIII of the coagulation cascade, but directly induces platelet aggregation and activation of the endothelium [2]. Moreover, it cleaves fibrinogen to fibrin, thus supporting the formation of a clot through fibrin polymerization. Meanwhile, the gelatin granules, which enable the material to fit to any irregular wound shape, swell and put pressure on the site of bleeding. Hemostatic agents are particularly effective at controlling bleeding from micro-vessels and can conform to irregular surfaces by virtue of their hydrophilic properties. Although in regular hemostasis the body is able to counteract clotting via release of antithrombotic molecules, such as anti-thrombin, Proteins $\mathrm{C}$ and $\mathrm{S}$, and tissue factor pathway inhibitor, intravascular embolization of large amounts of thrombin can produce devastating effects [3]. Several papers reported single cases or series of fatal pulmonary embolism in different surgical context, such as head and neck plastic surgery, neurosurgery or orthopedics [4-7]. This is to the best of our knowledge the first report of this adverse reaction in laparoscopic surgery.

\section{Case Report}

Here, we describe a patient with pulmonary micro-embolism associated with TBHM use in the management of hemostasis in videolaparoscopic cholecystectomy.

A 63 year-old male patient was scheduled for elective videolaparoscopic cholecystectomy in our General Surgery Ward. His anamnesis was positive for chronic hypertension successfully treated with olmesartan $20 \mathrm{mg}$, kidney-ureteral chronic stones previously treated with ultrasound extracorporeal lithotripsy and positioning of double J stent, and traumatic brain injury with loss of consciousness two years earlier. The patient had quit smoking since forty years and had no history of drug, alcohol or coffee abuse. Cholelithiasis was an occasional report of an abdomen echo tomography performed during a previous hospital admission subsequent to the mentioned traumatic brain injury. Indication to laparoscopic cholecystectomy was given after the patient suffered an episode of pain in the upper right quadrant of the abdomen radiating to the right shoulder. A new echo tomography confirmed the previous finding of multiple gallbladder stones, with associated cholecistitis. The patient's general conditions were optimal, his body mass index was 24 and no other risk factor for thrombophilia was detected. Standard prophylaxis for thromboembolism with low weight heparin was prescribed and administered 12 hours before surgery and elastic compression leg bandage was positioned right before the procedure. Induction of anesthesia was uneventful. The operation lasted 80 minutes and was uncomplicated, pneumoperitoneum never exceeded $12 \mathrm{mmHg}$ and hemodynamic and respiratory parameters were stable throughout the procedure. The only difficulty was represented by the important fibrosis in the hepatobiliary triangle and cystic bed that slowed down the procedure. To ensure a proper hemostasis the surgeon was bound to cover the cystic bed with a matrix of gelatin and human thrombin (FlosealTM). There had been no visible leakage of an arterial or venous vessel, and FloSeal was applied as indicated by the producer's instructions [8]. Five minutes after awakening from anesthesia, the patient developed abrupt dyspnea with no signs of airway obstruction, severe anxiety and thoracic oppression unresponsive to oxygen therapy. Saturation dropped to $85 \%$ despite 3 to 5 liters of oxygen administered through facemask. Tachycardia and mild hypertension developed (110 beats per minute and $160 / 100 \mathrm{mmHg}$ ). An arterial blood gas analysis showed respiratory and metabolic acidosis with lactate $2.6 \mathrm{mmol} / \mathrm{l}$

*Corresponding author: Paolo Mura, Department of Medical Sciences and Public Health, University of Cagliari, Cagliari, Italy, Tel: +39 070 6756533; E-mail: mura_paolo@hotmail.com

Received: February 20, 2018; Accepted March 05, 2018; Published March 12, 2018

Citation: Mura P, Fakir S, Mura S, Onida P, Finco G (2018) Non-Fatal Thrombin Based Hemostatic Matrix Pulmonary Embolism in Laparoscopic Surgery. A Case Report. J Pharmacovigil 6: 255. doi:10.4172/2329-6887.1000255

Copyright: ( $) 2018$ Mura P, et al. This is an open-access article distributed under the terms of the Creative Commons Attribution License, which permits unrestricted use, distribution, and reproduction in any medium, provided the original author and source are credited. 
Citation: Mura P, Fakir S, Mura S, Onida P, Finco G (2018) Non-Fatal Thrombin Based Hemostatic Matrix Pulmonary Embolism in Laparoscopic Surgery. A Case Report. J Pharmacovigil 6: 255. doi:10.4172/2329-6887.1000255

Page 2 of 3

with hypoxemia: $\mathrm{p}^{\mathrm{H}} 7.3$, $\mathrm{p}^{\mathrm{CO} 2} 51 \mathrm{mmHg}, \mathrm{p}^{\mathrm{O} 2} 65 \mathrm{mmHg}$, base excess-2,3 $\mathrm{mmol} / \mathrm{L}$. Electrocardiography was negative for myocardial ischemia and thoracic radiography was negative for pneumomediastinum, pneumothorax or parenchimal lesions. To rule out abnormalities in the pulmonary circulation, a thoracic angiotomography was requested and performed. The image showed multiple scattered bilateral minus images of the segmental and sub segmental branches of the pulmonary artery (Figures 1-3). The diagnosis of peripheral pulmonary embolism (PE) was confirmed by the radiologist and by the cardiology consultant as the clinic suggested. A second blood gas analysis performed four hours after the development of the symptoms, showed an improvement of the respiratory failure ( $\mathrm{p}^{\mathrm{O} 2} 94 \mathrm{mmHg}$ with oxygen $3 \mathrm{l} / \mathrm{min}$ and $\mathrm{p}^{\mathrm{CO} 2}$ $44 \mathrm{mmHg}$ ), along with a slight increase in lactate concentration (3.3 $\mathrm{mM})$. An echo tomography of the lower limbs failed to demonstrate any alteration of deep venous circulation. The symptoms receded spontaneously during the next six hours. A subsequent thoracic angiotomography after 96 hours showed a complete resolution of the previous circulation defects (Figures 4-6). The patient was released to his home after the second angiothomography. Recovery from surgery was uneventful. No further therapy was prescribed.

\section{Discussion}

We present the first evidence of a possible association between the use of hemostatic agents and PE in patients following videolaparoscopic cholecystectomy in a man who received intra-operative TBHM for hemostasis in video-laparoscopic cholecystectomy. The relationship between use of these agents and development of system thromboembolic events remains poorly understood. Reported adverse events include thromboembolism causing acute right heart failure with disseminated intravascular coagulation through inadvertent intravascular injection [9] and antibody formation against bovine factor $\mathrm{V}$ leading to life-threatening hemorrhage [10].
In a 2001 study that investigated the circulatory response of intravascular applied bovine and human thrombin in swine, all animals survived the application of the maximal dose of human thrombin (60 $\mathrm{U} / \mathrm{kg}$ ), whereas 5 of 6 animals with an equal dose of bovine thrombin died of hypotension and disseminated intravascular coagulation [11].

In 2012 a case of TBHM embolism of the left pulmonary artery was diagnosed by autopsy after in surgery for the removal of a spinal schwannoma [5]. Another case report showed a fatal intraoperative cardiac arrest after application of TBHM into a bleeding iliac screw defect [6]. A case of bilateral PE was ascribed to intraoperative use of thrombin-based hemostatic matrix following lumbar spine interbody fusion [12]. Development of a PE was also reported in an 18-year-old woman after intraoperative use of TBHM with unintended intravascular introduction of the material [9]. A series of five cases reports that there may be an association between the use of haemostatic matrix in head and neck oncologic reconstructions and pulmonary embolism [4].

In a retrospective study of approximately 467 patients undergoing meningioma resection, a positive relationship between the use of hemostatic matrix and development of VTE was identified. Interestingly, the use of anticoagulation was not linked with a reduction of VTE in this study [2]. A retrospective, multicenter, clinical review showed that incidence of thromboembolic events after use of gelatin-thrombinbased hemostatic matrix during intracranial tumor surgery is more frequent in patients who have been given a volume of TBHM greater than $10 \mathrm{ml}$ [11]. On the opposite a retrospective cohort study regarding the use of hemostatic agent during laparoscopic hysterectomy showed an increased predicted rate of blood transfusions $(\mathrm{P}=0.019)$, pelvic abscess diagnoses $(\mathrm{P}=0.001)$, hospital re-admission $(\mathrm{P}=0.001)$, and re-operation $(\mathrm{P}=0.021)$ but did not mentioned any increased risk of thromboembolic events [13]. A prospective case-control study in total hysterectomy surgery failed to find significant differences in terms of

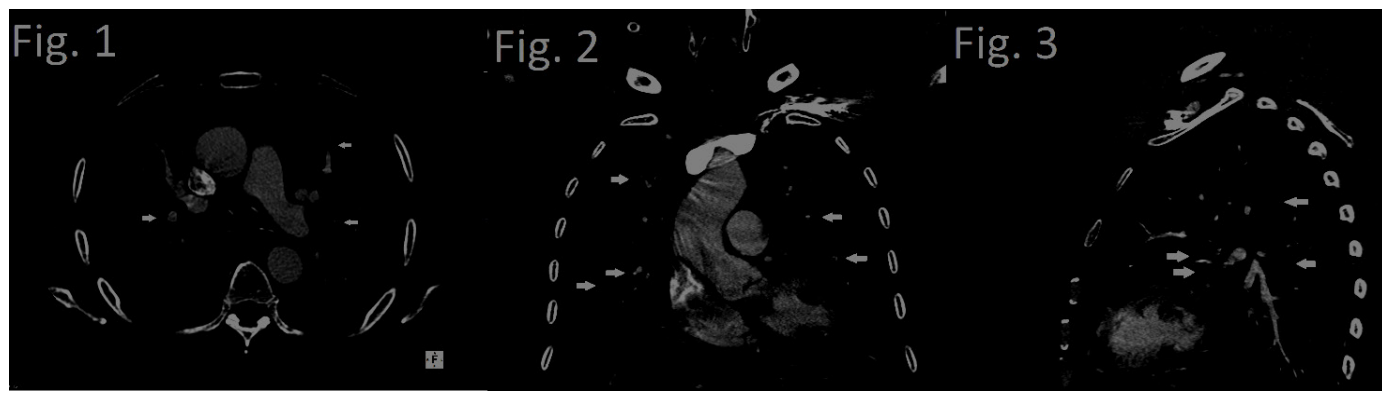

Figures 1-3: Radiographic diagnosis with angio-CT: In axial (Figure 1), coronal (Figure 2) and sagittal (Figure 3) sections, multiple minus-images in the segmental branches of pulmonary arteries are suggestive for non-massive bilateral pulmonary embolism. Main branches are not affected.

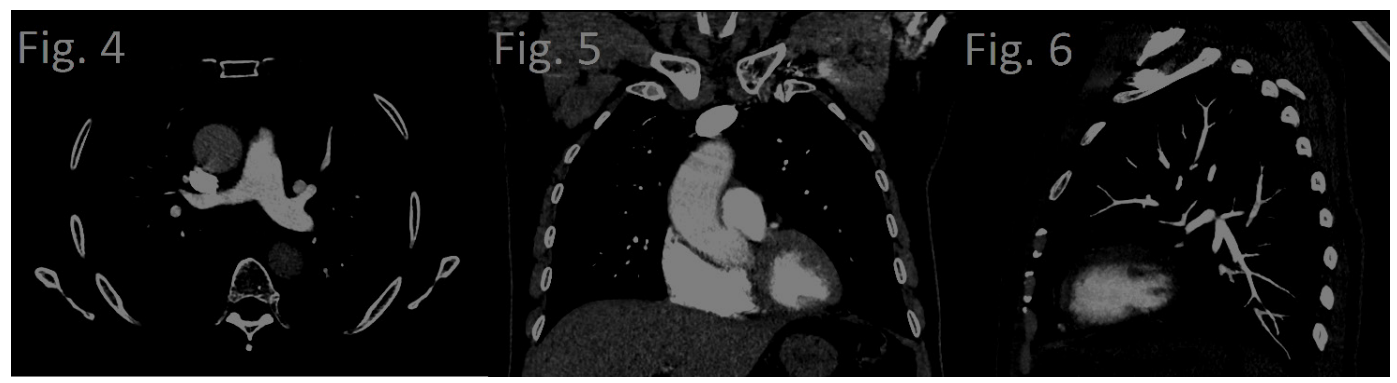

Figures 4-6: Angio-CT radiological control after $96 \mathrm{~h}$ : The absence of the previous findings in axial (Figure 4), coronal (Figure 5) and sagittal (Figure 6) sections indicate a complete resolution of the thromboembolic event. 
postoperative complications associated with TBHM use [13]. A metaanalysis suggested that the use of thrombin-based hemostatic agents in total knee arthroplasty is not related to the adverse reactions or complications such as wound infection or thromboembolic events [7]. No DVT was noted on the lower extremity duplex stud, and that does not completely rule out a DVT, but it makes it very unlikely: as matter of fact $28 \%$ of patients with PE have no identifiable thrombembolic source [14]. Furthermore no sign of portal venous thrombosis were detected during surgery, such splenomegaly, esophageal or gastric varices, ascites or other signs of portal hypertension and not even risk factors for hypercoagulability states. So it is unlikely that any microemboli could have detached and cross the portal system.

\section{Conclusion}

We report for the first time the case of a patient with bilateral PE associated with TBHM use in video-laparoscopic cholecystectomy. As TBHM is a widely used intraoperative hemostatic agent, surgeons and anesthesiologists should be aware of the potential risk of TBHMassociated PE. Our experience indicates that common pharmacological prophylaxis, such as subcutaneous heparin, is likely ineffective in reducing occurrence of $\mathrm{PE}$ in the setting of TBHM use. The relationship between use of these agents and development of systemic thromboembolic events is still not clearly stated despite the increasing number of reports suggesting this possible outcome.

Although we cannot definitively attribute these events to use of hemostatic matrix, there is biologic rationale to explore the association. The temporal relationship of PE formation to the index surgery, the presence of multiple bilateral thromboembolism, the absence of any signs of deep venous thrombosis, the previous administration of low molecular weight heparin, the application of leg elastic bandage and the short time length of surgery, along with the absence of any risk factor for hypercoagulability, further support a TBHM-related PE. It is likely that TBHM granules migrated through small undetected open vessels in the cystic bed to the systemic venous circulation leading to a PE. Despite being a rare adverse reaction, TBHM embolism after laparoscopic surgery should be considered possible, even when the device is used according to the producer's instructions, and advises for proper monitoring of patients in the immediate postoperative period when used.

\section{References}

1. Oz MC, Rondinone JF, Shargill NS (2003) FloSeal Matrix: New generation topical hemostatic sealant. J Card Surg 18: 486-493.

2. Safaee M, Sun MZ, Oh T, Aghi MK, Berger MS, et al. (2014) Use of thrombinbased hemostatic matrix during meningioma resection: A potential risk factor for perioperative thromboembolic events. Clin Neurol Neurosurg 119:116-20.

3. Chapman WC, Singla N, Genyk Y, McNeil JW, Renkens KL Jr, et al.(2007) A phase 3, randomized, double-blind comparative study of the efficacy and safety of topical recombinant human thrombin and bovine thrombin in surgical hemostasis. J Am Coll Surg 205:256-265.

4. Yue BYT, Zinn R, Roberts R, Wilson J (2017) Use of thrombin-based haemostatic matrix in head and neck reconstructions: A potential risk factor for pulmonary embolism. ANZ J Surg 87: E276-E280.

5. Steinestel K, Geiger A, Naraghi R, Kunz U, Danz B, et al. (2013) Fatal thromboembolism to the left pulmonary artery by locally applied hemostatic matrix after surgical removal of spinal schwannoma: A case report. Hum Pathol 44: 294-298.

6. Skovrlj B, Motivala S, Panov F, Steinberger J, Stuelpnagel J, et al.(2014) Fatal intraoperative cardiac arrest after application of surgifoam into a bleeding iliac screw defect. Spine 39: 1239-1242.

7. Wang C, Han Z, Zhang T, Ma JX, Jiang X, et al. (2014) The efficacy of thrombin-based hemostatic agent in primary total knee arthroplasty: A metaanalysis. J Orthop Surg Res 9: 90.

8. Baxter Healthcare FloSeal: Instructions for application. Zurich, Switzerland: Baxter Healthcare SA, 2011.

9. Ferschl MB, Rollins MD (2009) Thromboemboli, acute right heart failure and disseminated intravascular coagulation after intraoperative application of a topical hemostatic matrix. Anesth Analg 108: 434-436.

10. Cheng CM, Meyer-Massetti C, Kayser SR (2009) A review of three stand-alone topical thrombins for surgical hemostasis. Clin Ther 31: 32-41.

11. Pusateri AE, Holcomb JB, Bhattacharyya SN, Harris RA, Gomez RR, et al. (2001) Different hypotensive responses to intravenous bovine and human thrombin preparations in swine. J Trauma 50: 83-90.

12. Wei Z, Elder BD, Goodwin CR, Witham TF (2015) Bilateral pulmonary emboli associated with intraoperative use of thrombin-based hemostatic matrix following lumbar spine interbody fusion. J Clin Neurosci 22: 1502-1505.

13. Harris JA, Uppal S, Kamdar N, Swenson CW, Campbell D, et al. (2017) A retrospective cohort study of hemostatic agent use during hysterectomy and risk of post-operative complications. Int J Gynaecol Obstet 136: 232-237.

14. Schwartz T, Hingorani A, Ascher E, Marks N, Shiferson A, et al. (2012) Pulmonary embolism without deep venous thrombosis. Ann Vasc Surg 26: 973976. 